\title{
Proteomic landscape of drug-tolerant persister subpopulation of Mycobacterium tuberculosis
}

Rishabh Sharma ${ }^{\#}$, Ajitesh Lunge ${ }^{\#, \$}$, Nikita Mangla\#, and Nisheeth Agarwal ${ }^{\star}$

\section{Affiliation:}

\#Translational Health Science and Technology Institute, NCR Biotech Science Cluster, 3rd Milestone, Faridabad-Gurgaon Expressway, Faridabad- 121001 (Haryana)

\$Jawaharlal Nehru University, New Mehrauli Road, New Delhi- 110067 (Delhi) ${ }^{*}$ Correspondence author: Nisheeth Agarwal, E-mail: nisheeth@thsti.res.in

\section{Table of contents}

Page S-2: Fig. S1. Validation of differentially expressed proteins by immunoblotting. Page S-3: Fig. S2. Comparative analysis of transcript and protein levels of persister genes.

Page S-4: Fig. S3: Organization of genes encoding persister proteins.

Dataset S1. Quantitative proteomic analysis of Mtb persisters by iTRAQ-MS/MS (Excel file)

Dataset S2. Analysis of differentially expressed proteins in persister subpopulation of Mtb H37Rv (Excel file) 
Figure S1: Validation of differentially expressed proteins by immunoblotting. (*) represents blot regions that are presented in Figure 4. Shown are immunoblots of whole cell lysates of Mtb after 2- and 16 days of growth under the in vitro model of persistence. Immunoblotting was performed with anti-HtpG (top), anti-YidC (middle) and anti-ClpP2 (bottom) antibodies, respectively. Blots were developed by using enhanced chemiluminescence substrate. The Western blots comprise of the entire membrane which is cut into three portions at the respective positions, and probed individually with the respective antibodies. L, Pre-stained protein ladder; 2D, samples prepared on day 2; 16D, samples prepared on day 16.

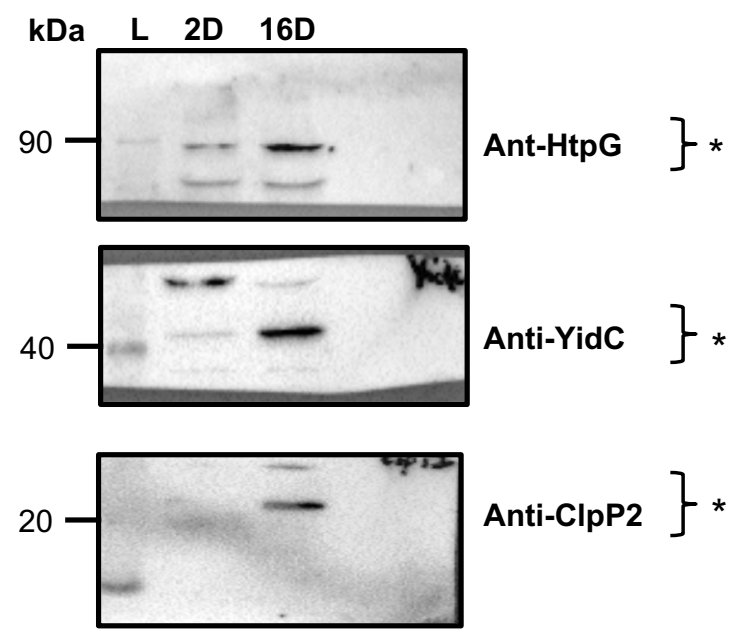


Figure S2: Comparative analysis of transcript and protein levels of persister genes in Mtb. Shown is the fold-change in the transcript and protein levels of persister genes in Mtb after 16 days of growth under the in vitro model of persistence. While transcript levels were estimated by RT-PCR, the protein levels were determined by ITRAQ-based quantitative proteomics. Mean values from two biological experiments \pm s.d. are shown.

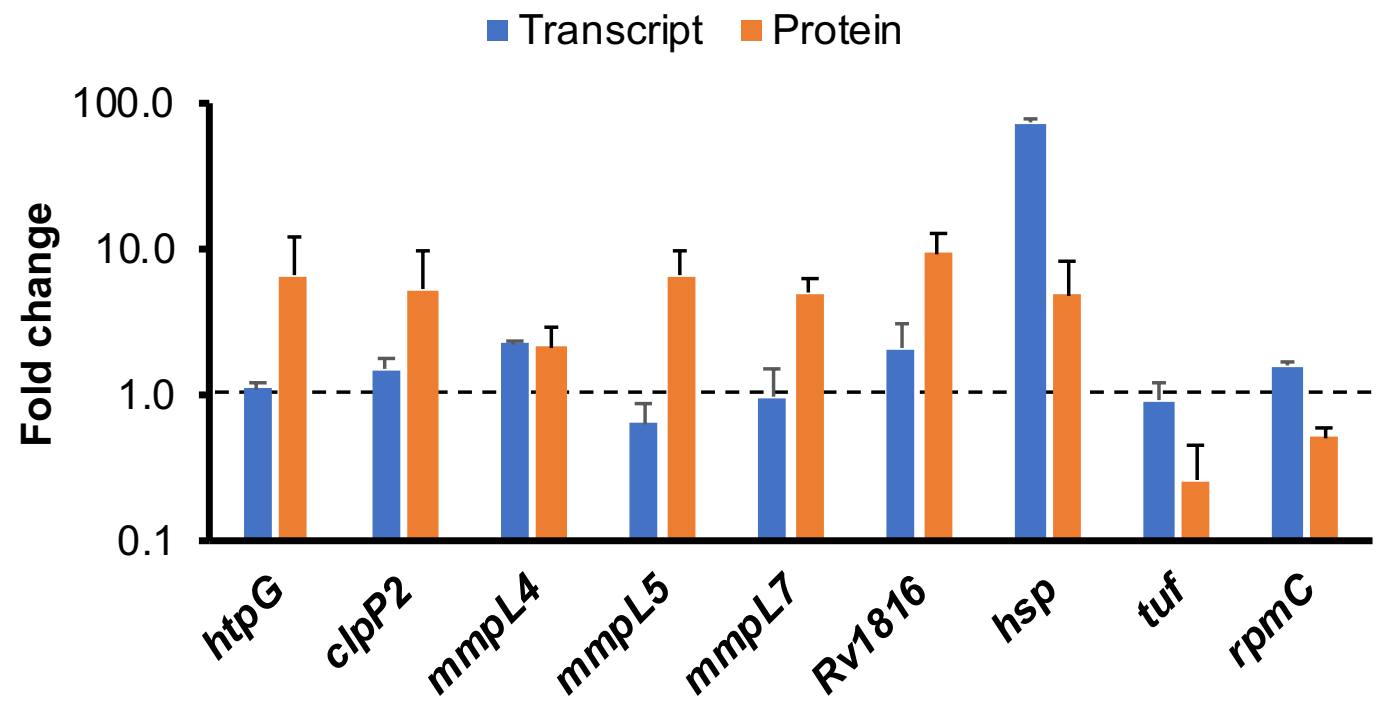


Figure S3: Organization of genes encoding persister proteins. Shown are various genes encoding persister proteins that are predicted to be organized into operon. Operon prediction was performed using MicrobesOnline Operon Prediction tool (http://www.microbesonline.org/cgi-bin/fetchLocus.cgi?locus=31772).

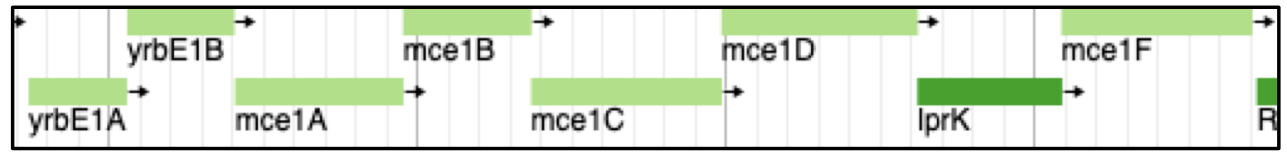
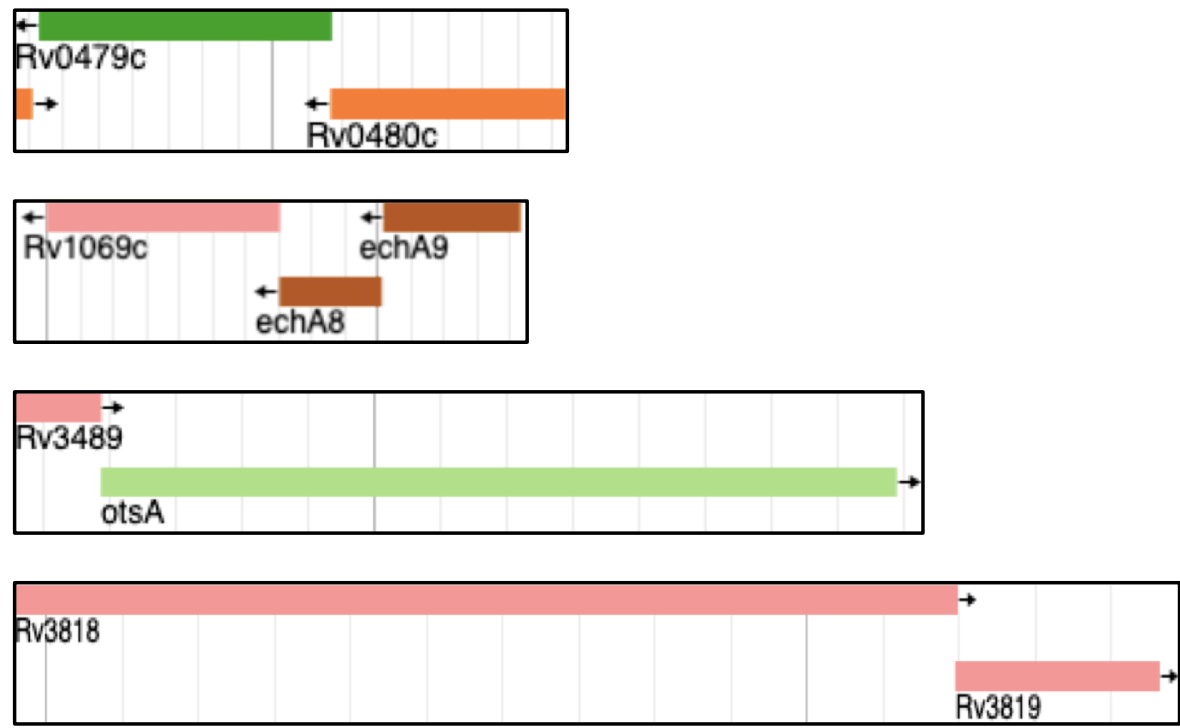